\title{
Channel Assignment in Virtual Cut-Through Switching based Wireless Mesh Networks
}

\author{
Dola Saha, Aveek Dutta, Dirk Grunwald and Douglas Sicker \\ University of Colorado \\ Boulder, CO 80309-0430 USA
}

\begin{abstract}
Conventional wireless networks employ a contention based channel access mechanism, which not only imposes high latency but also reduces goodput of the network. Lack of interference estimation algorithms over the entire network results in unpredictable collision, packet loss and retransmissions. Advances in multicarrier modulation techniques enable us to group subcarriers into orthogonal subchannels and treat them separately as information carriers. While this provides an increased number of non-interfering channels, intelligent utilization of the given spectrum is also required. In this paper, a solution for decreasing latency in mesh networks has been proposed by aptly incorporating a virtual cut-through switching technique to route packets in the network. To alleviate the impact of interference on packet reception, we also propose a fast pair-wise interference detection scheme, which is used for channel allocation. The cumulative performance of the proposed protocol shows improvement over existing Wi-Fi based mesh networks that provide a motivating platform for future protocol developments using this technique.
\end{abstract}

\section{Introduction}

Orthogonal Frequency Division Multiplexing (OFDM) is a multicarrier modulation scheme that has the ability to transmit data in closely spaced multiple carriers that do not interfere with each other. Using OFDM as the basic technology, Orthogonal Frequency Division Multiple Access (OFDMA) has been developed as a multi-user channel access mechanism that combines a subset of the available subcarriers into a subchannel, which then can be used for multiple access techniques. Orthogonal subchannels can be utilized for simultaneous transmission and reception by any node equipped with two antennas, one for transmission and the other for reception, provided the transmission and reception subchannels do not overlap. Such an approach minimizes the latency of each packet, and thus motivates the investigation of intelligent allocation of subchannels to nodes for simultaneous transmission and reception.

This paper focuses on utilization of OFDMA for concurrent transmission and reception such that each node in a wireless network has a property to relay packets "on the fly" while it is still receiving a part of the packet. To efficiently relay packets to non-interfering subchannels, we propose a centralized channel allocation mechanism, which utilizes the information of link and possible interference. The link information is gathered by transmitting a known training sequence. The interference information is also generated by scheduling two transmissions at the same time, and detecting the 
actual interference. Then, this link and interference information is converted to an edgecoloring problem of graph theory, where only chosen routes are considered for channel assignment.

We evaluate our protocol through simulations and experiment with a varied set of topologies to show improvement in various parameters. We also propose a FPGA based prototype hardware platform which is capable of relaying packets from one subchannel to another. Our evaluation suggests that with correct detection of possible interference in the network and proper utilization of this information for channel allocation algorithms leads to encouraging performance enhancements for wireless networks.

\section{Related Work}

Inter-nodal processing delay plays an important role in determining the overall performance of the network. Researchers have made constant efforts to decrease this latency to improve the network throughput. Ram Ramanathan [1] proposes to bypass the inadequacies of the conventional IP stack to support Wireless Mesh Networks by using virtual cut-through circuit switching thus reducing the overhead of four-way handshaking. Mctasney et al. [2, 3] introduce wireless wormhole switching to minimize the processing delay in each node by aptly incorporating the idea of virtual channels in flit-based wormhole switching techniques [4] in multi-computer networks. Our proposed protocol has lower latency than abovementioned methods, and we compare them in more details in 3.2

To efficiently utilize the potential of wireless virtual cut-through switching, we need to define resource allocation mechanisms. Ramanathan [5] proposes to unify the scheme of channel assignment in varied architectures into a generalized graph coloring method, which we utilize to assign subchannels to the relay nodes in our network. However, these techniques are developed for platforms not capable of simultaneous transmission and reception as a relay.

Interference in the wireless medium is a key contributor to packet loss, higher latency, retransmissions and control overheads. For a practical virtual cut-through switching without any MAC layer acknowledgment, we need good knowledge of the source of interference. Most of the previous work in this domain [6-8] is based on interference estimation rather than actual measurement. In this paper, we propose to measure and detect the possible interference before channel assignment, which enhances the overall performance of the network.

\section{Wireless Virtual Cut-Through Switching}

\subsection{Physical Layer Architecture}

Utilizing the OFDMA technique, a node is expected to simultaneously transmit data packets generated at its own application layer, receive data packets that are destined to its application layer and also forward or relay data packets from one subchannel to another. To perform these tasks simultaneously, a node is equipped with two antennas, one for transmission and another for reception. The node initially receives the signal 
from the receiver antenna, performs Fast Fourier Transform (FFT) to convert the time domain signal to frequency domain. The signal is then passed through the equalizer block to compensate for any phase or amplitude errors introduced as it passes through the channel. Next, the signal is passed through the channel switch block, which is set by the channel assignment algorithm to switch the frequency to a different subchannel. Finally, the signal is converted back to time domain by the Inverse Fast Fourier Transform (IFFT) block and transmitted by the transmitter antenna. If the relaying node has packets to transmit, it has to be fed into the IFFT block at the same time as the switched, relayed signal, but at a different subchannel. However, if the channel switch block does not know the number of the subchannel to relay, then the complete packet is received, decoded and forwarded to MAC layer of the relaying node.

\subsection{Comparison with Concurrent Technologies}

Figure 1 shows approximate latency incurred in four systems, IEEE 802.11, flit-based relay, Time Division Multiplexing(TDMA) and virtual cut-through switching. It has to be noted, that IEEE 802.11 has a half duplex radio, and so there is no overlapping transmissions. Also, there is significant amount of gap in between the packets due to back-off algorithms. When we move from IEEE 802.11 to flit-based systems, which has a full-duplex radio, we have to divide the whole bandwidth into three to overlap the transmissions for all the three hops. Hence, the transmission time of any packet is increased three times. If $T$ is the time required to transmit a packet using full bandwidth, then the time required to transmit the same packet in one-third of the bandwidth is $3 T$. The processing delay is added in each of the flits, which increases as we opt for smaller flit size. Time Division Multiple Access can be utilized to minimize the flit overhead, where the whole packet is transmitted all at once, using the complete bandwidth, but the transmissions shall not overlap in time, which will cause interference. The virtual cut through switching utilizes partial bandwidth for transmission in each hop and switches to a different part of the bandwidth in the next hop. It has an advantage of transmitting a complete packet all together, and hence the processing delay is incorporated once in the packet. The latency clearly shows that using wireless virtual cut-through switching, a packet is transferred to destination in minimum time.

There are a few assumptions in the physical layer, which we expect that with advancement of research and technologies, will be feasible in the near future. Our primary assumption is that all the nodes are time synchronized with a precision of less than a microsecond, such that all the OFDM symbols at any receiver overlap in time. We only consider co-channel interference, and no adjacent-channel interference, which may occur at the relay due to close spacing of the transmitting and receiving subcarriers. Research on various interference cancellation techniques [9, 10] may lead to reduction of co-channel interference in our cut-through switching.

\section{Receiver and Interference Detection at Physical Layer}

\subsection{Challenges of Interference Detection}

The existing wireless networks aim to minimize interference and thus collision by either transmitting control packets like RTS and CTS and thus reserving the transmission 


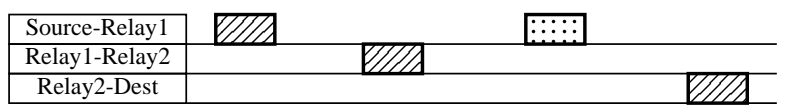

IEEE 802.11 [Packet TxTime $=\mathrm{T}]$

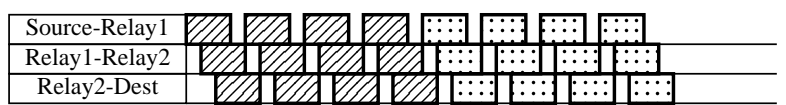

Flit-based Relay, [Packet TxTime $=3 \mathrm{~T}$, Flit TxTime $=3 \mathrm{~T} / 4$ ]

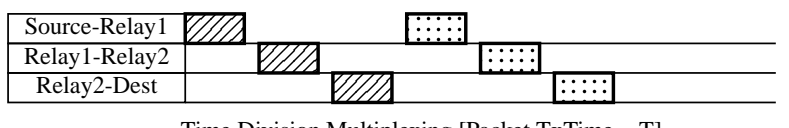

Time Division Multiplexing [Packet TxTime $=\mathrm{T}]$

\begin{tabular}{|c|c|c|c|}
\hline \multicolumn{4}{|l|}{ Source-Relay1 } \\
\hline Relay1-Relay2 & & & \\
\hline Relay2-Dest & 7211 & : & \\
\hline
\end{tabular}

Virtual Cut-Through Switching [Packet TxTime = 3T]

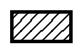

Packet 1 or Part of Packet 1

Packet 2 or Part of Packet 2

Fig. 1. Comparison of Latency of Packets - X Axis showing time

medium, or by sensing the channel. We show that none of these techniques are appropriate for proper estimation of interference in the wireless channel. Figure 2$]$ shows a simple network of five nodes. Consider that there is an ongoing communication from node N4 to node N3. If we consider that nodes N3 and N4 have negotiated transmissions by RTS and CTS mechanism, then all nodes in the area within the transmission range of node $\mathrm{N} 3$ and $\mathrm{N} 4$, are aware of the communication and will not transmit until the transmission from node $\mathrm{N} 4$ to $\mathrm{N} 3$ ends. Node $\mathrm{N} 2$ is however eligible to transmit since it has not received any RTS or CTS. But, the interference range of node N2 (shown by densely dotted circle), is much more than its transmission range. So, if it starts transmission, transmission of $\mathrm{N} 2$ will interfere with reception at node $\mathrm{N} 3$, since $\mathrm{N} 3$ is within the interference range of node $\mathrm{N} 2$. If node $\mathrm{N} 2$ uses carrier sensing mechanism, where the carrier sensing range equals the interference range, $\mathrm{N} 2$ will still not be able to sense the signal from node $\mathrm{N} 4$, which is outside the interference range of $\mathrm{N} 2$. If the sensing range of $\mathrm{N} 2$ is extended to include node N4, shown in loosely dotted circle, N2 will actually sense the signal transmitted from node $\mathrm{N} 4$ and will wait to avoid interference. However, if the transmission from node N4 is not meant for node N3, but for node N5, then there will be no interference at node N5. But, increasing the sensing range will lead to unnecessary backoff and underutilization of the wireless media.

\subsection{Link Detection}

In this section, we propose a link detection scheme, which is utilized later in routing and channel assignment. The link detection scheme is similar to any "hello-packet" transmission scheme to detect the reception; but instead of transmitting packets, we transmit a series of known sequences of duration of one OFDM symbol. The symbol duration, 


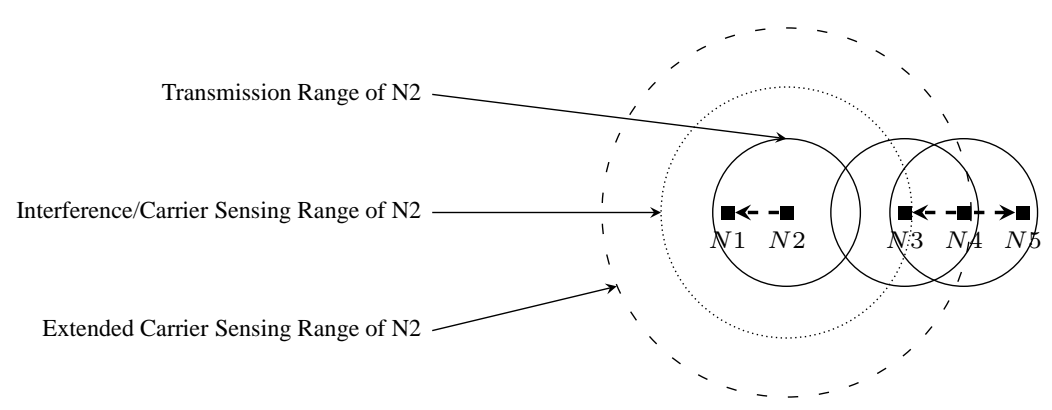

Fig. 2. Challenges of Interference Detection

denoted by $S$, is $4 \mu s$ in IEEE 802.11a [11], and hence the overhead of link detection is extremely low compared to packet transmission techniques. We utilize the existence of time synchronized network to schedule each node's transmission in each time slot of duration of one OFDM symbol. All other nodes except for the transmitter waits in receive mode and if the sequence is received correctly, the node recognizes the existence of the link $l_{i, j}$, where node $n_{i}$ is the transmitter and node $n_{j}$ is the receiver. However, for precise estimation of existing links, this procedure has to be done repeatedly for $X$ times, $X>1$. This procedure has to be performed periodically in a mobile environment to gather the neighborhood information. However, it has to be noted that the link detection scheme considers asymmetric links, and so if $l_{i, j}$ is a valid link, that does not necessarily mean $l_{j, i}$ is also a valid link in the network.

\subsection{Interference Detection}

In this section, we propose an interference detection scheme, which is utilized in the channel assignment algorithm to allocate interference-free subchannels to flows for routing packets. We exploit the existence of time synchronous nodes to schedule a pair of nodes to transmit at each time slot. As described in 4.2 we use known sequences of OFDM symbol duration to be transmitted by the transmitters. All other nodes wait in receiving mode to receive the sequence. A receiver can (a) possibly receive the sequence without any interference, (b) may receive a garbled signal due to interference, or (c) may not receive any signal at all. We consider that in the first case, one of the transmitters is close enough to the receiver such that there is no interference from the other one and the link information already exists from the link detection phase. In the second scenario, there exists an interference, and the receiver node, $n_{k}$, recognizes the interference $i n t f_{i, j, k}$, which indicates that when nodes $n_{i}$ and $n_{j}$ transmit at the same time in same channel, they interfere at node $n_{k}$. In the last scenario, the transmitters are far away and do not interfere at node $n_{k}$. To eliminate any false detection, this procedure has to be done repeatedly $X$ times, $X>1$. This procedure has to be performed periodically in a mobile environment to gather the neighborhood information. 


\subsection{Overhead}

In this section, we discuss the overhead incurred in the link detection and interference detection scheme proposed in sections 4.2 and 4.3 The total time to detect all links in a network of $N$ nodes is given by $(N \times X \times S)$. The total time increases linearly as the number of nodes in the network increases. The total time to detect all possible interference in a network of $N$ nodes is given by $\left[\frac{n \times(n-1)}{2} \times X \times S\right] \mu$ s. The total time increases as a square of the nodes as the number of nodes in the network increases. In a 100 node scenario with $X=2$, link detection and interference detection times are $0.8 \mathrm{~ms}$ and $40 \mathrm{~ms}$, respectively. But with approximate knowledge of geographic location, we can simultaneously scheduling multiple pairs for transmitters that are far away to essentially reduce the overhead.

\section{Interference-Aware Channel Assignment}

In this section, we propose the centralized channel assignment algorithm which takes advantage of the knowledge of existing link and interference information, and incorporates that into an edge coloring problem to allocate subchannels to links. Firstly, we assign subchannels to the links only where there is a possibility of packet exchange. We consider that the flows are predefined and we choose a shortest path from source to destination for each flow. So, the links that appear in the route are the only possible sites for packet exchange. By restricting the subchannel assignment only to the routes, we minimize the degree of complexity of the channel assignment problem. We also enhance the spectrum utilization by not assigning subchannels to any unused links.

Let us consider that $N$ is the set of all nodes in the network, and $n_{i}$ denotes the $i$-th node in the network. $L$ is the set of all valid links $l_{t, r}$ in the network, where $l_{t, r}$ denotes a link from node $n_{t}$ to $n_{r}$. In other words, if node $n_{t}$ transmits, then node $n_{r}$ receives the packet correctly in absence of any interference. $R$ is the set of all links that is a part of any route defined by the shortest path routing. Hence, $R \subseteq L$. I is the set of interference, consisting of $i n t f_{t_{1}, t_{2}, r}$, which indicates that when nodes $n_{t_{1}}$ and $n_{t_{2}}$ transmit at the same time, then the signals interfere at the node $n_{r}$.

Vertex Selection for Graph Coloring Problem We are interested in assigning subchannels to the links in the set $R$. Hence, we choose all the links $l_{t, r} \in R$ as the vertices in graph coloring problem. If a link has multiple occurrences in the set $R$, we incorporate all the occurrences as different vertices of the graph. Hence, the total number of vertices in the graph equals $|R|$.

Edge Selection for Graph Coloring Problem After selecting the vertices, we connect them with edges only where the vertices in the graph cannot be assigned the same color, or in other words, the links in the network cannot be assigned the same subchannel. With this notion in mind, we incorporate three procedures for edge inclusion.

Common Node - Select an edge between the vertices $l_{t_{1}, r_{1}}$ and $l_{t_{2}, r_{2}}$, if there exists a common transmitter or receiver in between these two links. This ensures that a relay 
node does not transmit and receive in the same subchannel to avoid interference at its reception. By assigning an edge between common transmitters (for example, $l_{t_{i}, r_{1}}$ and $l_{t_{i}, r_{2}}$ ) we ensure that separate subchannels are assigned to cater different flows handled by the same node $n_{t}$. Due to similar reasons, we assign an edge between common receivers. Mathematically, an edge is chosen if,

$\forall l_{t_{1}, r_{1}}, l_{t_{2}, r_{2}} \in R\left[\right.$ if $\left(n_{t_{1}}=n_{t_{2}}\right)$ or $\left(n_{t_{1}}=n_{r_{2}}\right)$ or $\left(n_{r_{1}}=n_{t_{2}}\right)$ or $\left.\left(n_{r_{1}}=n_{r_{2}}\right)\right]$. The algorithm for this procedure is shown in line 6 of algorithm 1

Link - If there exists a link between a transmitter and a receiver in the chosen routes, then they cannot be allocated the same subchannel, as the transmission from the transmitter will interfere with the reception at the receiver. This idea is incorporated to select another set of edges for the graph coloring problem. Mathematically, an edge is chosen if, $\forall l_{t_{1}, r_{1}}, l_{t_{2}, r_{2}} \in R\left[\right.$ if $\left(l_{t_{1}, r_{2}} \in L\right)$ or $\left.\left(l_{t_{2}, r_{1}} \in L\right)\right]$. The algorithm for this procedure is shown in line 9 of algorithm 1

Interference - Finally, we incorporate the edges between interfering links. From section 4.3. we are aware of the interference sites, and already generated set $I$ of interference. Hence, we examine all the links in the set $R$ in pairs, and choose an edge, if there exists an interference at any one of the two receivers when both the transmitters in the paired link transmit at the same time. Mathematically, an edge is chosen if, $\forall l_{t_{1}, r_{1}}, l_{t_{2}, r_{2}} \in R\left[\right.$ if $\left(i n t f_{t_{1}, t_{2}, r_{1}} \in I\right)$ or $\left.\left(i n t f_{t_{1}, t_{2}, r_{2}} \in I\right)\right]$. The algorithm for this procedure is shown in line 12 of algorithm 1

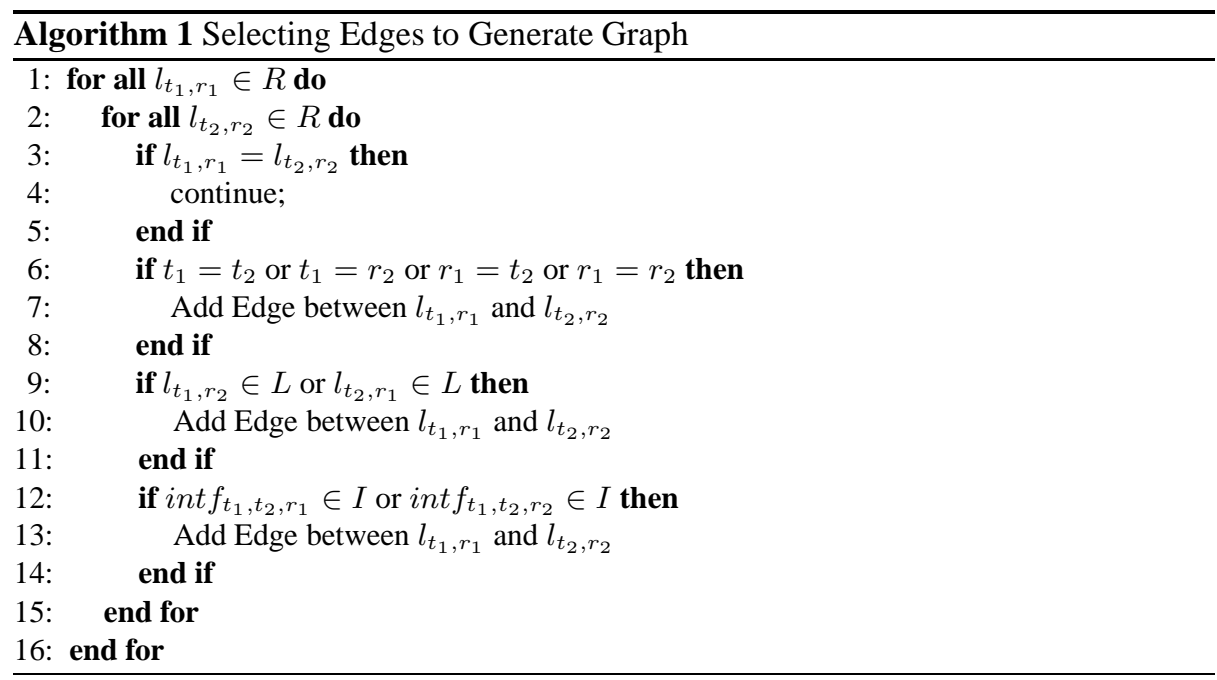

Graph Coloring Once the vertices and edges are selected for graph coloring problem, we use Progressive Minimum Neighbors First (PMNF) algorithm [5] to color the vertices of the graph. The graph coloring results in assignment of colors to the vertices in the graph, or assignment of subchannels to the links. The total number of subchannels required equals the total number of colors required for coloring. So, we divide the 
complete bandwidth of 48 subcarriers by the number of subchannels required to get the width of each subchannel. If 48 is not divisible by the number of subchannels, (for example 10), we utilize subcarriers upto the multiple of number of subchannels less than the total number of subcarriers (in this case 40). Although the bandwidth remains partially unutilized, we argue that this is required for fair allocation of bandwidth per flow.

\section{Hardware Implementation}

To demonstrate the proposed physical layer routing we implemented a prototype using a software defined radio platform. The SDR involves an OFDM transceiver, the design and implementation of which has been detailed in [12, 13]. The platform is capable of transmitting and receiving generic $802.11 \mathrm{a} / \mathrm{g}$ packets as described in physical layer specification [14]. The OFDM transceiver components consist of a custom radio frontend responsible for up/down conversion to/from the $2.4 G H z$ ISM band and a Xilinx ExtremeDSP development kit IV manufactured from Nallatech, containing Virtex-IV FPGA.

The OFDM receiver has been customized to be able to decode the information bits from a certain set of subcarriers, called subchannels. To make the transmission frequency agile, the $20 \mathrm{MHz}$ channel is split into two subchannels, subchannel \#1 has subcarriers -26 to -1 and subchannel \#2 has subcarriers 1 to 26 . This type of full duplex transceiver requires the use of two sets of radio front-ends. Using the receiver and the transmitter as a pipeline with negligible turnaround time between the receive and transmit mode, latency in multihop mesh networks can be significantly reduced. The pipeline is used to switch incoming packets on-the-fly onto another subchannel.

The spectrum of the incoming signal and the operation of the switch is shown in Fig 3 The top figure shows the incoming signal having data on all subcarriers. The second one shows that only information contained in subchannel \#2 has been separated and equalized. This signal is fed to the frequency switch and switched over to subchannel \#1 as shown in the third figure, followed by transmission by the transmitter front-end.

\section{Performance Evaluation}

To evaluate the performance of the proposed protocol, we performed simulations using QualNet [15] network simulator and compared the performance with conventional IEEE 802.11a based MAC protocol. Our protocol is referred to as 'relay', while IEEE 802.11a based MAC protocol is refereed to as '802.11' throughout the rest of the section. We implemented an OFDMA based transceiver in QualNet, operating at $2.4 \mathrm{GHz}$. The link detection and interference detection has also been implemented with $X=10$, such that 10 OFDM symbols were used to transmit and detect any link or interference. In order to make the simulation similar to the hardware under development [12, 13], we incorporated the processing delay at three stages: a) Transmission Delay equals to 

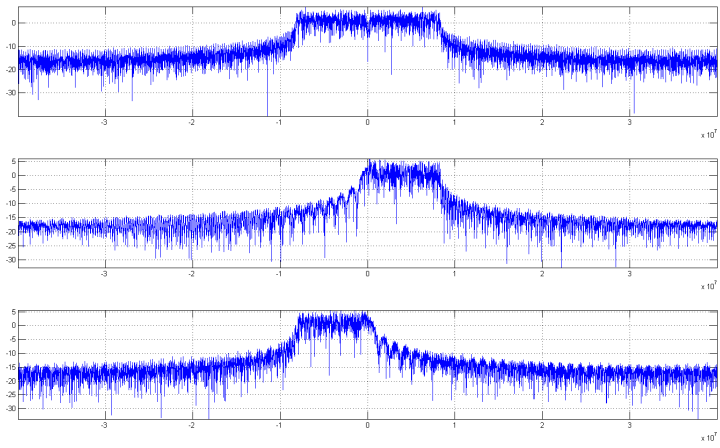

Fig. 3. Input and Output Spectrum for Switch

$11 \mu s$, b) Delay at Relay equals to $18 \mu \mathrm{s}$, and c) Reception Delay equals to $15 \mu \mathrm{s}$, . Table 1 shows the parameters used for simulation. We evaluate the protocol in random scenarios, varying the number of flows, and varying the number of nodes.

Table 1. Simulation Parameters

\begin{tabular}{|l|l|}
\hline Seeds & 10 \\
\hline Packet Size & 1024 bytes \\
\hline Simulation Time & 120 secs \\
\hline Pathloss Model & Two-Ray \\
\hline Application Layer & CBR \\
\hline Transport Layer & UDP \\
\hline Mobility & None \\
\hline Topology & Random \\
\hline CBR Packet Injection Interval & $615 \mu \mathrm{s}$ \\
\hline
\end{tabular}

Performance Evaluation with Increasing Number of Flows We increment the number of flows and evaluate the performance of our protocol, as shown in figure 4 Average throughput and PDR decreases, while end-to-end delay increases, as the number of flows increase in the network. This is due to more number of simultaneous transmission being scheduled at the same time to accommodate the increasing number of flows. In all the cases, 'relay' performs better than '802.11'. Also, we observe that the jitter shown in performance is much more noticeable in '802.11' than in 'relay'. The aggregate throughput of the network also improves up to 1.83 times in 'relay' over '802.11'.

Performance Evaluation with Increasing Number of Nodes We increment the number of nodes, keeping the node density constant and evaluate the performance of the 


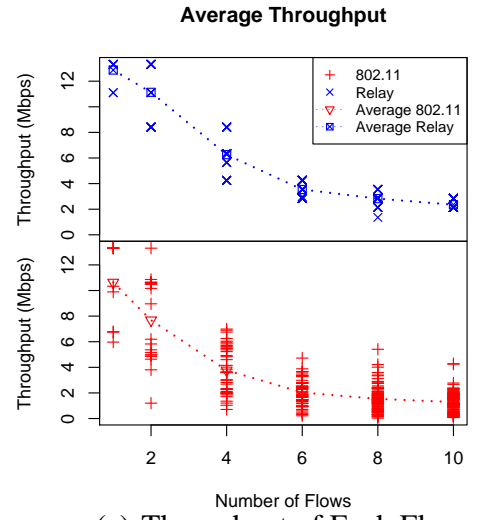

(a) Throughput of Each Flow

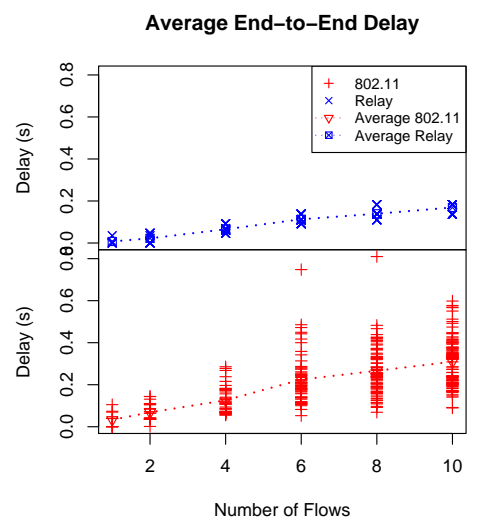

(c) Average End-to-End Delay per Flow

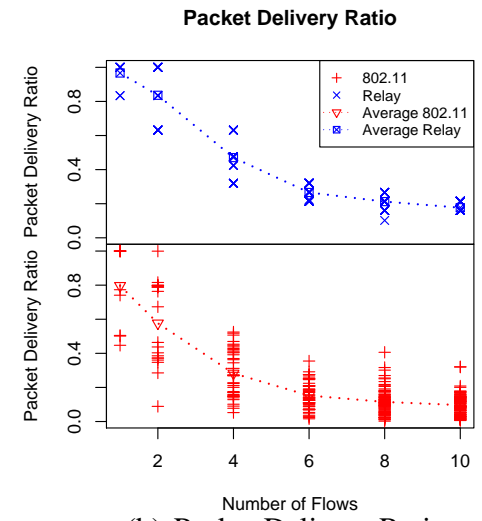

(b) Packet Delivery Ratio

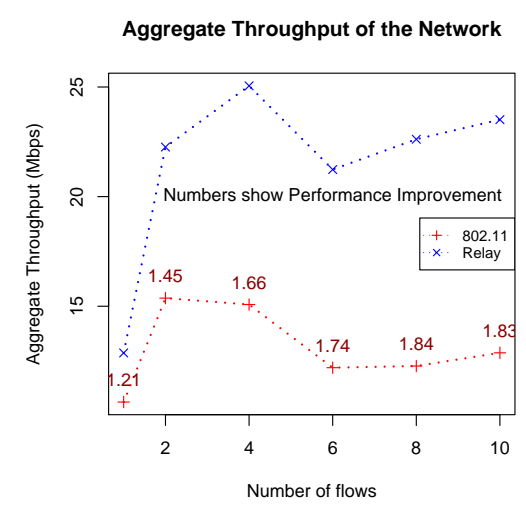

(d) Aggregate Throughput

Fig. 4. Performance Improvement in Random Scenario with Increasing Number of Simultaneous Flows

proposed protocol as shown in figure 5] Average throughput and PDR decreases, while end-to-end delay increases, as the number of nodes increase in the network. As the number of nodes increases, with node density being constant, the total area also increases, which indicates that the average hops for random source destination pairs also increases. With increasing number of hops, performance decreases due to more number of simultaneous communications in both ' 802.11 ' and 'relay'. In all the cases, 'relay' performs better than ' 802.11 '. Also, we observe that the jitter shown in performance is more in ' 802.11 ' than in 'relay'. The aggregate throughput of the network also improves up to 2.93 times in 'relay' over ' 802.11 '. The performance improvement is dependent on the topology, node density, number of flows and many other topological parameters, but on an average in most of the cases, we observe a two-fold improvement in overall performance of the network. 


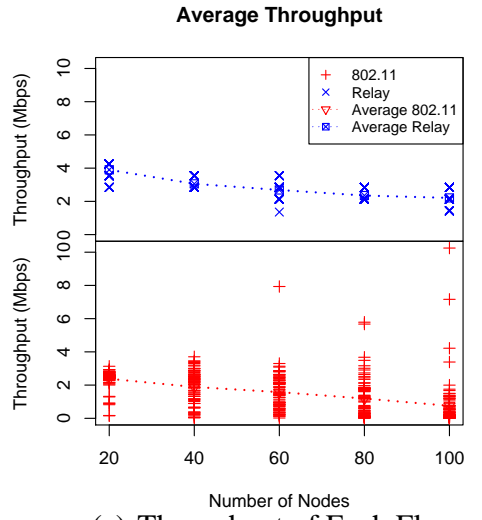

(a) Throughput of Each Flow

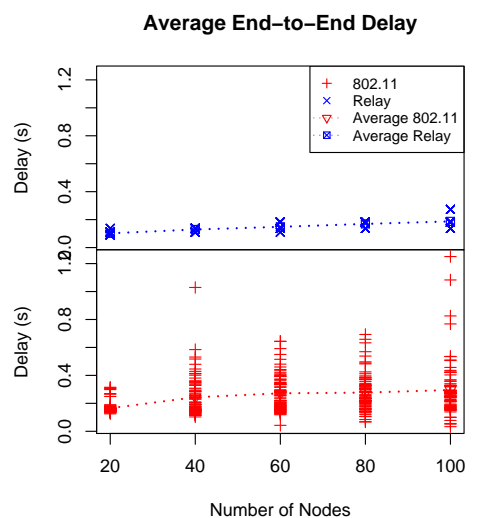

(c) Average End-to-End Delay per Flow

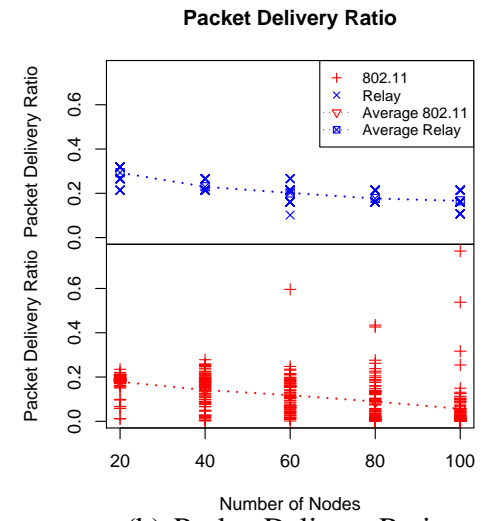

(b) Packet Delivery Ratio

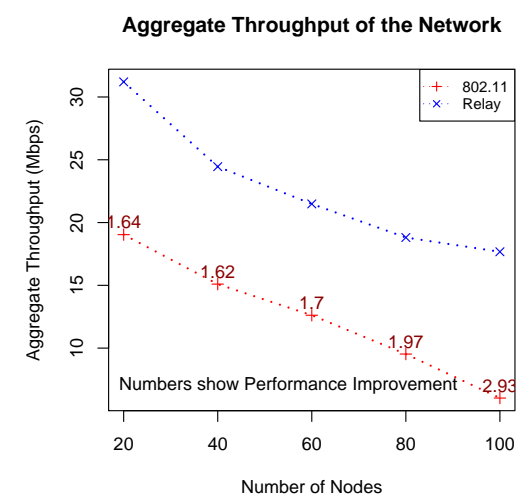

(d) Aggregate Throughput

Fig. 5. Performance Improvement in Random Scenario with Increasing Number of Nodes

\section{Conclusion}

In this paper, we investigate the challenges of interference detection in wireless networks and propose a solution for this. We also incorporate this detection information in a centralized channel assignment algorithm, that collects the information from all the nodes and processes the information centrally. Although much work remains to be done, this paper shows the potential of effective interference detection scheme and its use in channel assignment in virtual cut-through switching based wireless networks. The performance is improved with respect to increased average throughput, packet delivery ratio and end-to-end delay. The aggregate throughput of the network increases up to three times compared to the conventional IEEE 802.11 based MAC protocol. Based on our overall evaluation, we believe that the virtual cut-through switching based physical layer can offer significant performance improvements in wireless multihop networks. 


\section{Bibliography}

[1] Ramanathan, R., Tchakountio, F.: Channel access over path segments for ultra low latency manets. In: IEEE MILCOM 2007. (October 2007) 2007.

[2] McTasney, R., Grunwald, D., Sicker, D.: Low-Latency Multichannel Wireless Mesh Networks. In: Proceedings of the 16th International Conference on Computer Communications and Networks 2007 ICCCN 2007, New York, NY, USA, IEEE (2007) 1082-1087

[3] McTasney, R., Grunwald, D., Sicker, D.: Multichannel Wormhole Switching vs. CSMA/CA for Wireless Mesh Networking. In: Proceedings of the IEEE Wireless Communications and Networking Conference 2008 WCNC 2008, New York, NY, USA, IEEE Communications Society (2007)

[4] Dally, W.J., Seitz, C.L.: Deadlock-free message routing in multiprocessor interconnection networks. IEEE Trans. Comput. 36(5) (1987) 547-553

[5] Ramanathan, R.: A unified framework and algorithm for channel assignment in wireless networks. Wireless Networks 5(2) (1999) 81-94

[6] Zhang, X., Liu, Q., Shi, D., Liu, Y., Yu, X.: An average link interference-aware routing protocol for mobile ad hoc networks. In: ICWMC '07: Proceedings of the Third International Conference on Wireless and Mobile Communications, Washington, DC, USA, IEEE Computer Society (2007) 10

[7] ElBatt, T., Andersen, T.: Cross-layer interference-aware routing for wireless multi-hop networks. In: IWCMC '06: Proceedings of the 2006 international conference on Wireless communications and mobile computing, New York, NY, USA, ACM (2006) 153-158

[8] Sen, A., Ganguly, S.M.S., Bhatnagar, S.: An interference-aware channel assignment scheme for wireless mesh networks. In: Communications 2007. (2007)

[9] Halperin, D., Anderson, T., Wetherall, D.: Taking the sting out of carrier sense: interference cancellation for wireless lans. In: MobiCom '08: Proceedings of the 14th ACM international conference on Mobile computing and networking, New York, NY, USA, ACM (2008) 339-350

[10] Radunovic, B., Gunawardena, D., Proutiere, A., Singh, N., Balan, V., Key, P.: Efficiency and fairness in distributed wireless networks through self-interference cancellation and scheduling. Technical report, Microsoft Research (March 2009)

[11] 802.11a 1999, I.S.: Part 11: Wireless lan medium access control (mac) and physical layer (phy) specifications high-speed physical layer in the $5 \mathrm{ghz}$ band

[12] Fifield, J., Kasemir, P., Grunwald, D., Sicker, D.: Experiences with a platform for frequency-agile techniques. In: DYSPAN 2007. (2007)

[13] Dutta, A., Fifield, J., Schelle, G., Grunwald, D., Sicker, D.: An intelligent physical layer for cognitive radio networks. In: WICON '08: Proceedings of the 4th international conference on Wireless internet. (2008)

[14] 802.11-1999, I.S.: Part 11: Wireless lan medium access control (mac) and physical layer (phy) specifications

[15] Technologies, S.N.: QualNet network simulator, version 4.0 